\title{
Some Developments on Cup Anemometer Aerodynamics
}

\author{
Santiago Pindado ${ }^{a}$ and Javier Cubas ${ }^{b}$ \\ Instituto IDR/UPM, ETSIA, Universidad Politécnica de Madrid, Pza. Cardenal Cisneros 3, 28040 \\ Madrid. Spain
}

Keywords: cup anemometer; anemometer calibration, MEASNET, anemometer wear and tear, rotation analysis

\begin{abstract}
In the present study the geometry of cups is experimentally studied through anemometer performance. This performance is analyzed in two different ways. On the one hand the anemometer transfer function between cases is compared. On the other hand the stationary rotation speed is decomposed into constant and harmonic terms, the comparison being established between the last ones. Results indicate that some cup shapes can improve the uniformity of anemometer rotation, this fact being important to reduce degradation due to ageing.
\end{abstract}

\section{Introduction}

Along the twentieth century the cup anemometer has become the most popular instrument to measure the wind speed. It combines both simplicity and accuracy and, at present, it is used widely in the wind energy sector for many different activities (from wind energy production forecast, to wind mill performance control).

Due to its work as a MEASNET center concerning the calibration of anemometers, there is a great interest at the IDR/UPM Institute in the behavior of cup anemometers. For this reason a review of more than 10000 calibrations performed at IDR/UPM started in 2009, focusing on the performance of commercial anemometers, the effect of the calibration accuracy on the AEP (Annual Energy Production) estimations [1], the effect of ageing on the anemometers' performance after calibration [2], and the effect of climatic conditions (changes of temperature, pressure and density) on the anemometer transfer function [3].

Once the basic aerodynamics of the anemometer rotor was described with simple models $[4,5,6]$, the interest of many researchers focused more on the non-stationary aerodynamics (that is, the overspeeding problem) $[7,8,9,10]$. However, the aforementioned simple models have been recently revised and new conclusions have arisen in order to correlate the aerodynamics of an isolated cup with the performance of the anemometer's rotor [11].

The transfer function of an anemometer is represented by the following expression:

$$
V=\mathrm{A} \cdot f+\mathrm{B}
$$

where $V$ is the wind speed, $f$ is the anemometer's rotation frequency output, and A (slope) and B (offset) are the calibration coefficients. This linear equation, which correlates the wind speed and the anemometer's output frequency [12] has to be defined by means of a calibration process. The aforementioned transfer function can be rewritten in terms of the anemometers' rotation frequency, $f_{r}$, instead of the output frequency $f$, as:

$$
V=\mathrm{A}_{r} \cdot f_{r}+\mathrm{B}
$$

where $\mathrm{A}_{r}$ is the result of multiplying the calibration constant $\mathrm{A}$ by the number of pulses per revolution given by the anemometer, $N_{p}$. The number of pulses is different depending on the anemometer's inner system for translating the rotation into electric pulses. Magnets-based systems give from 1 to 3 pulses per revolution, whereas opto-electronics-based systems normally give higher pulse rates per revolution, from 6 to 44 [1].

Today, the cup anemometer has a standardized configuration of three cups, as the 3-cup anemometer has become the most efficient solution when compared to the 4-cup anemometer. This 
fact makes the anemometer's rotational speed not uniform [13]. The rotational speed of a 3-cup anemometer, $\omega$, under a perfectly constant and uniform wind speed, can be decomposed along one turn into a constant term, $\omega$, plus a series of harmonic terms dependent of a frequency three times higher than the one related to the mentioned constant term, and its multiples:

$$
\omega(t)=\omega_{0}+\omega_{3} \sin \left(3 \omega_{0} t+\varphi_{3}\right)+\cdots+\omega_{3 n} \sin \left(3 n \omega_{0} t+\varphi_{3 n}\right)+\cdots .
$$

However, it should be also pointed out that for practical terms these harmonic terms are not taken into account as a contribution to the rotational speed. Analyzing the information from [13] with regard to a 3-cup anemometer rotational speed measured in one turn, it can be verified that the second term of Eq. 3, that is, the third harmonic coefficient of this rotational speed Fourier series decomposition, is only $7.8 \%$ of the constant term (this third harmonic term being the highest one), see Fig. 1.

In the present study the cups' geometry is experimentally studied through anemometer performance. This performance is analyzed in two different ways. On the one hand the anemometer transfer function is compared from one another case between different cases. On the other hand the stationary rotation speed is decomposed into constant and harmonic terms, the comparison being established between the harmonic terms. Results indicate that some cup shapes can improve the uniformity of anemometer rotation, this fact being important to reduce degradation due to ageing.
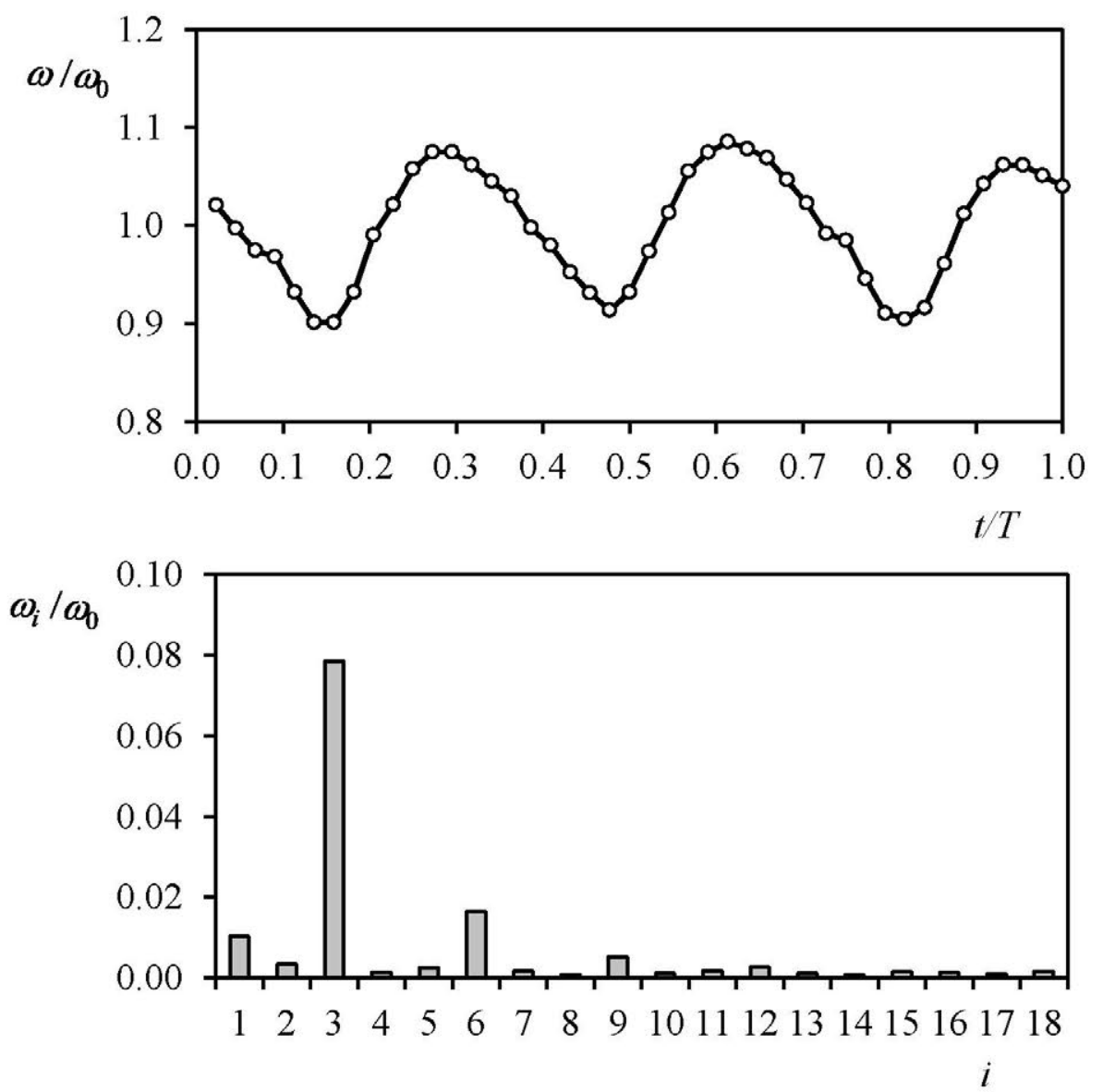

Figure 1. Relative-to-the-average rotational speed, $\omega / \omega_{0}$, of a Thies anemometer during one turn at $8 \mathrm{~m} / \mathrm{s}$ wind speed [13] (top), and non-dimensional values of the Fourier series decomposition performed on that rotational speed, $\omega_{i} / \omega_{0}$ (bottom). 


\section{Testing Configuration and Calibration Setup}

The Ornytion 107A anemometer was used in the present study. 10 different rotors were tested (see Table 1 and Figs. 2 and 3): 4 were equipped with conical cups $\left(90^{\circ}\right.$ cone-angle, all with the same cup radius: $R_{c}=25 \mathrm{~mm}$, with the cup center rotation radius (defined in Fig. 3) varying from $R_{r c}=40 \mathrm{~mm}$ to $R_{r c}=100 \mathrm{~mm}$ ). Three were equipped with elliptical cups (front surface equal to the conical cups: $S_{c}=1963.5 \mathrm{~mm}^{2}$ and $R_{r c}=60 \mathrm{~mm}$ ). Three were equipped with porous cups (front surface, including the empty area, equal to the conical cups: $S_{c}=1963.5 \mathrm{~mm}^{2}$, cup radius: $R_{c}=25$ $\mathrm{mm}$, truncated shape with hole diameter $h=9 \mathrm{~mm}, h=19 \mathrm{~mm}$, and $h=24 \mathrm{~mm}$, and $R_{r c}=60 \mathrm{~mm}$ ). Calibrations were performed in the calibration wind tunnel at the Mechanical Engineering Department at the Vrije Universiteit Brussel, Belgium. The calibrations were carried out following MEASNET procedures [14]. The analog output signal of the anemometer during the calibrations performed on every rotor was sampled at 30 seconds at $5000 \mathrm{~Hz}$. This provides enough number of points to perform an accurate Fourier analysis of the signal and isolate the third harmonic coefficient.

Table 1. Geometrical characteristics of the rotors tested: cups' center rotation radius, $R_{r c}$, front area of the cups, $S_{c}$, cup radius (conical and porous cups), $R_{c}$, hole diameter of the porous cups, $h$, and semi-major and semi-minor axes, $a$ and $b$, of the elliptical cups. See also Fig. 2.

\begin{tabular}{|c|c|c|c|c|}
\hline \multicolumn{5}{|c|}{ Conical cups } \\
\hline Rotor & $\boldsymbol{R}_{\boldsymbol{c}}[\mathbf{m m}]$ & $\boldsymbol{S}_{\boldsymbol{c}}\left[\mathbf{m m}^{\mathbf{2}}\right]$ & $\boldsymbol{R}_{\boldsymbol{r c}}[\mathbf{m m}]$ & - \\
\hline $\mathrm{c}-25 / 40$ & 25 & 1963.5 & 40 & - \\
\hline $\mathrm{c}-25 / 60$ & 25 & 1963.5 & 60 & - \\
\hline $\mathrm{c}-25 / 80$ & 25 & 1963.5 & 80 & - \\
\hline $\mathrm{c}-25 / 100$ & 25 & 1963.5 & 100 & - \\
\hline \hline \multicolumn{5}{|c|}{ Elliptical cups } \\
\hline Rotor & $\boldsymbol{a}[\mathbf{m m}]$ & $\boldsymbol{b}[\mathbf{m m}]$ & $\boldsymbol{S}_{\boldsymbol{c}}\left[\mathbf{m m}^{\mathbf{2}}\right]$ & $\boldsymbol{R}_{\boldsymbol{r c}}[\mathbf{m m}]$ \\
\hline $\mathrm{a}-27 / 60$ & 27 & 23.15 & 1963.5 & 60 \\
\hline $\mathrm{a}-30 / 60$ & 30 & 20.83 & 1963.5 & 60 \\
\hline $\mathrm{a}-35 / 60$ & 35 & 17.86 & 1963.5 & 60 \\
\hline \multicolumn{5}{|c|}{ Porous cups } \\
\hline Rotor & $\boldsymbol{R}_{\boldsymbol{c}}[\mathbf{m m}]$ & $\boldsymbol{S}_{\boldsymbol{c}}\left[\mathbf{m m} \mathbf{2}^{\mathbf{2}}\right]$ & $\boldsymbol{R}_{\boldsymbol{r c}}[\mathbf{m m}]$ & $\boldsymbol{h}[\mathbf{m m}]$ \\
\hline $\mathrm{h}-09 / 60$ & 25 & 1963.5 & 60 & 9 \\
\hline $\mathrm{h}-19 / 60$ & 25 & 1963.5 & 60 & 19 \\
\hline $\mathrm{h}-24 / 60$ & 25 & 1963.5 & 60 & 24 \\
\hline
\end{tabular}
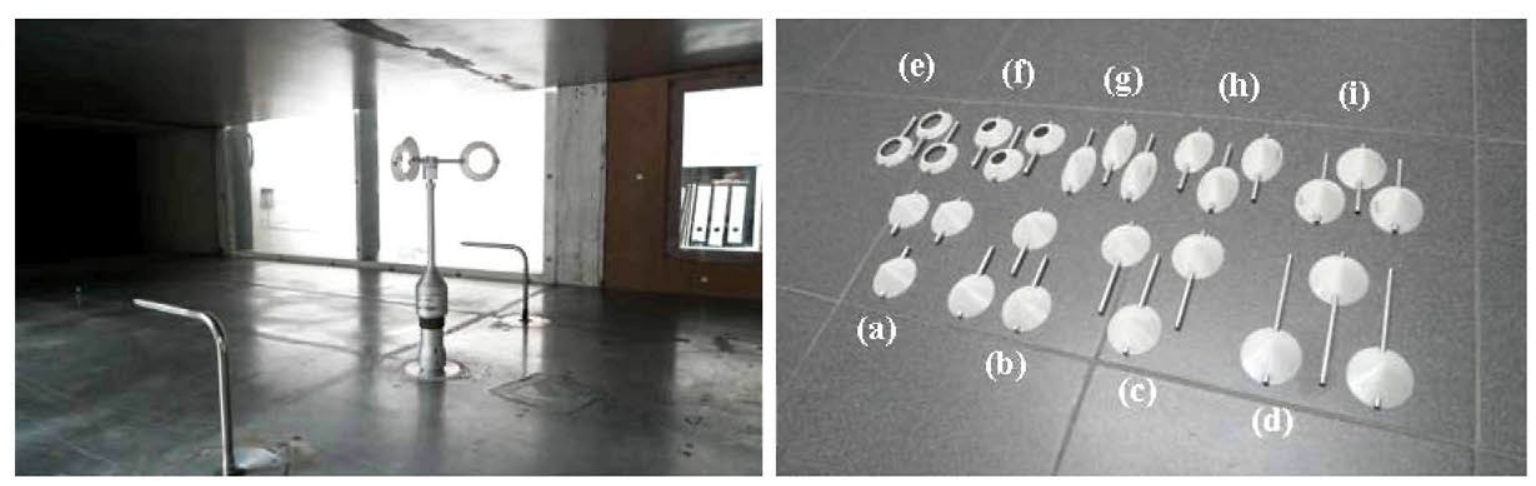

Figure 2. Left: Calibration wind tunnel at the Department of Mechanical Engineering of the Vrije Universiteit Brussel. Right: Different rotor cups measured: (a): c-25/40;
(c): c-25/80;
(d): c-25/100;
(e): h-24/60;
(f): h-19/60;
(g): a-35/60;
(i): a-27/60 (h-09/60 rotor cups are not shown in the picture).
(h): a-30/60;

(b): c-25/60; 

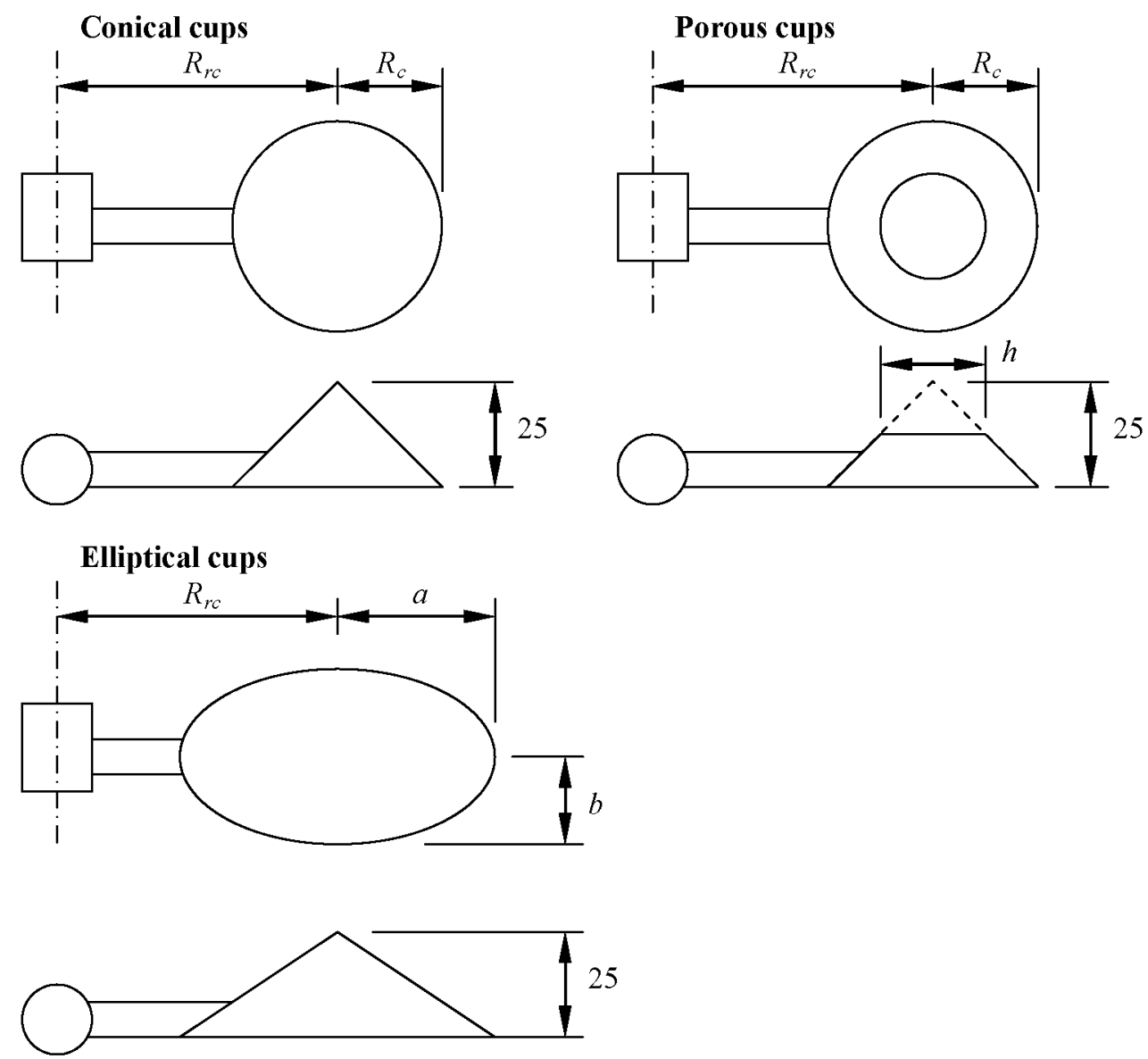

Figure 3. Sketch of the cups and rotor geometries tested. Dimensions in $\mathrm{mm}$. See also Table 1

\section{Results}

In Table 2 the results of the calibrations are shown. In order to make the effect of the rotor's shape on the anemometer performance more clear, the rotation frequency, $f_{r}$, has been calculated from these results at $10 \mathrm{~m} / \mathrm{s}$ wind speed, see Fig. 4 . It can be seen that, as expected, the rotation speed is increased if the cup center rotation radius, $R_{r c}$, is reduced, that is, shorter rotor arms increase the efficiency of the anemometer in transforming the energy from the wind flow into rotation. On the other hand, just comparing the effect of the cup shapes with the same cup center rotation radius, the aforementioned efficiency is obviously reduced increasing the porosity of the cups (reducing the aerodynamic drag coefficients). The same tendency is observed increasing the ratio $a / b$ of the elliptical cups' front area. Nevertheless, this effect is lower in comparison with the one related to the porous cups.

Table 2. Calibration constants from each rotor transfer function measured.

\begin{tabular}{||c|c|c|c|c|c||c|c|c||}
\hline \hline Rotor & $\mathbf{A}_{\boldsymbol{r}}[\mathbf{m}]$ & $\mathbf{B}[\mathbf{m} / \mathbf{s}]$ & Rotor & $\mathbf{A}_{\boldsymbol{r}}[\mathbf{m}]$ & $\mathbf{B}[\mathbf{m} / \mathbf{s}]$ & Rotor & $\mathbf{A}_{\boldsymbol{r}}[\mathbf{m}]$ & $\mathbf{B}[\mathbf{m} / \mathbf{s}]$ \\
\hline $\mathrm{c}-25 / 40$ & 0.9050 & 0.0828 & $\mathrm{~h}-09 / 60$ & 1.6567 & 0.2792 & $\mathrm{a}-27 / 60$ & 1.6116 & 0.1762 \\
\hline $\mathrm{c}-25 / 60$ & 1.5241 & 0.2425 & $\mathrm{~h}-19 / 60$ & 2.0018 & 0.5270 & $\mathrm{a}-30 / 60$ & 1.6288 & 0.1666 \\
\hline $\mathrm{c}-25 / 80$ & 2.1283 & 0.3471 & $\mathrm{~h}-24 / 60$ & 3.5077 & 0.4269 & $\mathrm{a}-35 / 60$ & 1.6625 & 0.1388 \\
\hline $\mathrm{c}-25 / 100$ & 2.7396 & 0.4379 & - & - & - & - & - & - \\
\hline
\end{tabular}




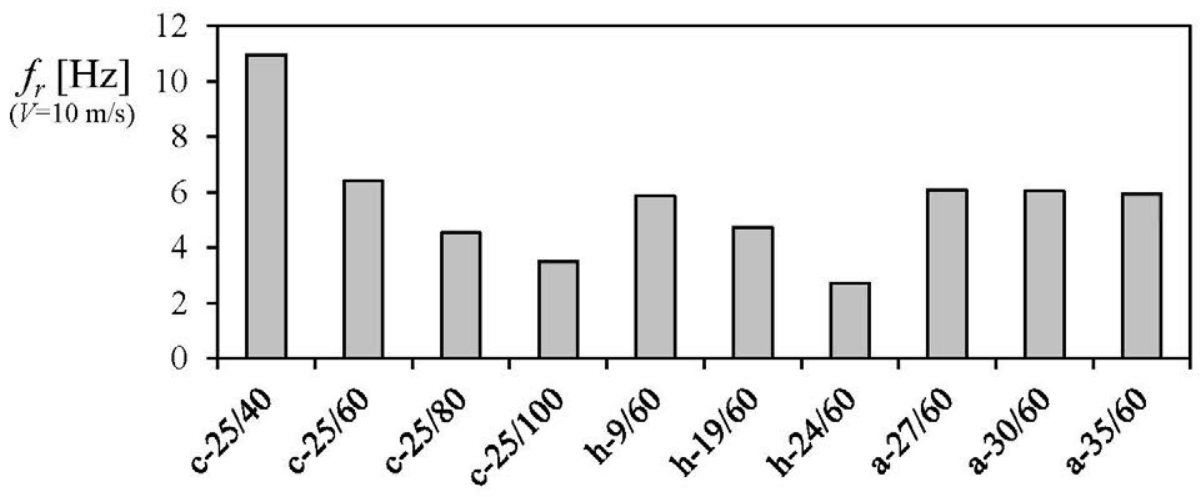

Figure 4. Rotation frequency, $f_{r}$, at $10 \mathrm{~m} / \mathrm{s}$ wind speed calculated from the calibration results (see Table 2), for each rotor tested.

An indirect procedure to estimate the third harmonic constant of the Fourier series decomposition has been developed, as the anemometer used in the testing campaign, the Ornytion $107 \mathrm{~A}$, gives an analog output consisting in two pulses per revolution. For each rotor and each wind speed tested, the following signal has been obtained for several periods of $T$ seconds ( $T$ being the rotation period in each case, $T=1 / f_{r}$ ):

$$
y^{\prime}(t)=y(t)-\Omega \sin \left(\frac{2 \pi}{T / 2} t+\varphi\right)
$$

where the second term represents a 2-pulse per turn signal with amplitude $\Omega$, this parameter being the amplitude of the anemometer's output signal $y(t)$, and finally, $\varphi$ being the phase angle that gives the better approach to the analog output, $y(t)$. The function $y^{\prime}(t)$ is then the result of filtering $y(t)$, extracting the highest harmonic term which represents the anemometer rotation, and it can be decomposed into Fourier series:

$$
y^{\prime}(t)=y_{1}^{\prime} \sin \left(\frac{2 \pi}{T} t+\zeta_{1}\right)+y_{2}^{\prime} \sin \left(2 \frac{2 \pi}{T} t+\zeta_{2}\right)+y_{3}^{\prime} \sin \left(3 \frac{2 \pi}{T} t+\zeta_{3}\right)+\cdots
$$

On the other hand, if the rotating magnetic field produced by a 2-pole rotor is:

$$
B(t)=A \sin (2 \theta(t)+\xi)
$$

where $\theta$ is the angular position of the rotor, then, according to the Lenz law the output signal of the anemometer is a voltage level proportional to the derivative of the magnetic flux:

$$
V(t)=-N \frac{\partial \phi}{\partial t}=-N S \frac{\partial B}{\partial t}=-N S A 2 \frac{\partial \theta}{\partial t} \cos (2 \theta(t)+\xi)
$$

where $N$ and $S$ are respectively the number of spires and the cross area of the anemometer's inductor. Introducing now the expression of the angular speed (Eq. 3), Eq. 7 turns into:

$$
V(t) \approx-k\left[\omega_{0} \cos \left(2 \omega_{0} t+\xi\right)+\omega_{3} \sin \left(3 \omega_{0} t+\varphi_{3}\right) \cos \left(2 \omega_{0} t+\xi\right)\right]
$$

where $k$ is proportional to $N, S$, and the amplitude of the magnetic field, $A$. Finally, the second term of the above expression can be developed in single harmonic terms:

$$
V(t) \approx-k\left[\omega_{0} \cos \left(2 \omega_{0} t+\xi\right)+\frac{\omega_{3}}{2} \cos \left(\omega_{0} t+\varphi_{3}-\xi\right)-\frac{\omega_{3}}{2} \cos \left(5 \omega_{0} t+\varphi_{3}+\xi\right)\right]
$$

As it can be observed, the first harmonic term of the output signal is proportional to the third harmonic coefficient of the anemometer's rotating speed, so the same happens with the filtered 
signal, $y^{\prime}(t)$. This procedure was applied in each case for several periods of rotation in order to obtain an averaged value of the first harmonic coefficient of $y^{\prime}(t)$ related to the module of the postprocessed signal, $y_{1}^{\prime} / \Omega$. The results are shown in Fig. 5 for each rotor and every wind speed tested. It can be observed that the third harmonic of the rotational speed is lower for shorter cup arms, this indicates lower minor accelerations during rotation that could reduce degradation (periodic recalibrations of anemometers used in the field due to wear and tear are very common in the wind energy industry). On the other hand the same effect has been observed comparing rotors with the same cup center rotation radius but different cup shapes: h-19/60 and a-35/60 rotors seem to have more uniform rotation speeds when compared to c-25/60 rotor.
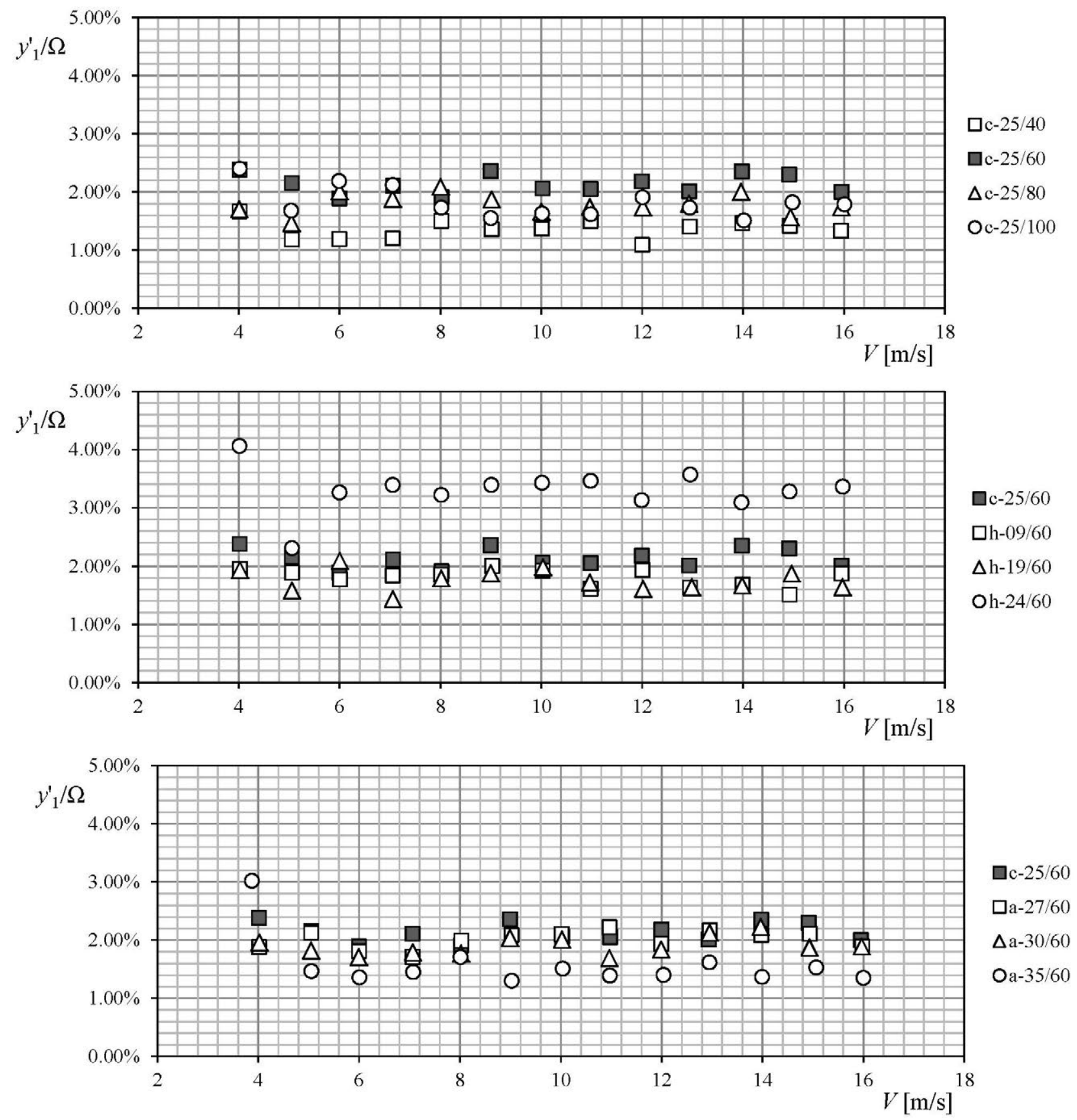

Figure 5. Percentage value of the first harmonic coefficient of the post-processed output signal referred to the module of the post-processed signal, $y_{1}{ }_{1} / \Omega$. 


\section{Conclusions}

In the present work the output signal of an anemometer tested with different rotor configurations has been analyzed. The comparison between configurations has been done through the analysis of the two parameters, the average rotation speed of the anemometers, and the third harmonic coefficient of their rotation speed. The major conclusions resulting from this study are:

- Lower values of the cups' center rotation radius tend to produce more efficient rotors in terms of transforming the wind speed into rotational speed.

- The rotation uniformity of a cup anemometer is related to the cups' center rotation radius. Rotors with lower values of this parameter tend to have more uniform rotational speed.

- The shape of the cups can also improve the rotation uniformity of an anemometer.

\section{References}

[1] S. Pindado, E. Vega, A. Martínez, E. Meseguer, S. Franchini, I. Sarasola, Analysis of calibration results from cup and propeller anemometers. Influence on wind turbine Annual Energy Production (AEP) calculations, Wind Energy 14 (2011) 119-132.

[2] S. Pindado, A. Barrero-Gil, A. Sanz, Cup anemometers' loss of performance due to ageing processes, and its effect on Annual Energy Production (AEP) estimates, Energies 5 (2012) 16641685 .

[3] S. Pindado, A. Sanz, A. Wery, Deviation of cup and propeller anemometer calibration results with air density, Energies 5 (2012) 683-701.

[4] M. Sanuki, S. Kimura, H. Obana, The performance of half-covered cup-anemometers. Papers in Meteorology and Geophysics, Meteorological Research Institute of Japan 14 (1963) 52-57.

[5] S. Ramachandran, A theoretical study of cup and vane anemometers, Q. J. R. Meteorol. Soc. 95 (1969) 163-180.

[6] J. Kondo, G. Naito, Y. Fujinawa, Response of cup anemometer in turbulence, J. Meteorol. Soc. Japan 49 (1971) 63-74.

[7] E.L. Deacon, The over-estimation error of cup anemometers in fluctuating winds, J. Sci. Instr. 28 (1951) 231-234.

[8] N.E. Busch, L. Kristensen, Cup anemometer overspeeding, J. Appl. Meteor. 15 (1976) 13281332 .

[9] J. C. Wyngaard, Cup, propeller, vane, and sonic anemometers in turbulence research, Annual Review of Fluid Mechanics 13 (1981) 399-423.

[10] R.S. Hunter, The accuracy of cup anemometer calibration with particular regard to testing wind turbines, Wind Engineering 14 (1990) 32-43.

[11] S. Pindado, J. Pérez, S. Avila-Sanchez, On cup anemometer rotor aerodynamics, Sensors 12 (2012) 6198-6217.

[12] L. Kristensen, Cup anemometer behavior in turbulent environments, Journal of Atmospheric and Oceanic Technology 15 (1998) 5-17.

[13] J.-A. Dahlberg, T.F. Pedersen, P. Busche, ACCUWIND -Methods for Classification of Cup Anemometers, Risø-R-1555 (EN), Risø National Laboratory, Roskilde, Denmark, 2006.

[14] Measuring Network of Wind Energy Institutes (MEASNET), Anemometer Calibration Procedure, Version 2, 2009. 